\title{
Lower bounds on geometric Ramsey functions*
}

\author{
MAREK ELIÁŠ ${ }^{\dagger}$ \\ Department of Applied Mathematics \\ Charles University, Malostranské nám. 25 \\ 11800 Praha 1, Czech Republic \\ Edgardo Roldán-Pensado \\ Institute of Theoretical Computer Science \\ ETH Zurich, 8092 Zurich, Switzerland
}

\author{
JiŘÍ MATOUŠEK \\ Department of Applied Mathematics \\ Charles University, Malostranské nám. 25 \\ 11800 Praha 1, Czech Republic, and \\ Institute of Theoretical Computer Science \\ ETH Zurich, 8092 Zurich, Switzerland \\ ZuZANA SAFERnOVÁt, \\ Department of Applied Mathematics and \\ Computer Science Institute \\ Charles University, Malostranské nám. 25 \\ 11800 Praha 1, Czech Republic
}

\begin{abstract}
We continue a sequence of recent works studying Ramsey functions for semialgebraic predicates in $\mathbb{R}^{d}$. A $k$-ary semialgebraic predicate $\Phi\left(x_{1}, \ldots, x_{k}\right)$ on $\mathbb{R}^{d}$ is a Boolean combination of polynomial equations and inequalities in the $k d$ coordinates of $k$ points $x_{1}, \ldots, x_{k} \in \mathbb{R}^{d}$. A sequence $P=\left(p_{1}, \ldots, p_{n}\right)$ of points in $\mathbb{R}^{d}$ is called $\Phi$-homogeneous if either $\Phi\left(p_{i_{1}}, \ldots, p_{i_{k}}\right)$ holds for all choices $1 \leq i_{1}<\cdots<i_{k} \leq n$, or it holds for no such choice. The Ramsey function $R_{\Phi}(n)$ is the smallest $N$ such that every point sequence of length $N$ contains a $\Phi$-homogeneous subsequence of length $n$.

Conlon, Fox, Pach, Sudakov, and Suk constructed the first examples of semialgebraic predicates with the Ramsey function bounded from below by a tower function of arbitrary height: for every $k \geq 4$, they exhibit a $k$-ary $\Phi$ in dimension $2^{k-4}$ with $R_{\Phi}$ bounded below by a tower of height $k-1$. We reduce the dimension in their construction, obtaining a $k$-ary semialgebraic predicate $\Phi$ on $\mathbb{R}^{k-3}$ with $R_{\Phi}$ bounded below by a tower of height $k-1$.

We also provide a natural geometric Ramsey-type theorem with a large Ramsey function. We call a point sequence $P$ in $\mathbb{R}^{d}$ order-type homogeneous if all $(d+1)$-tuples in $P$ have the same orientation. Every sufficiently long point sequence in general position in $\mathbb{R}^{d}$ contains an order-type homogeneous subsequence of length $n$, and the corresponding Ramsey function has recently been studied in several papers. Together with a recent work of Bárány, Matoušek, and Pór, our results imply a tower function of $\Omega(n)$ of height $d$ as a lower bound, matching an upper bound by Suk up to the constant in front of $n$.
\end{abstract}

\section{Introduction}

Ramsey's theorem and the classical Ramsey function. A classical and fundamental theorem of Ramsey claims that for every $n$ there is a number $N$ such that for every coloring

\footnotetext{
* Research supported by the ERC Advanced Grant No. 267165 and by ETH Zurich.

${ }^{\dagger}$ Partially supported by the Charles University Grants GAUK 1262213 and SVV-2013-267313.

${ }^{\ddagger}$ Partially supported by the project CE-ITI (GACR P202/12/G061) of the Czech Science Foundation.

${ }^{\S}$ Partially supported by the project CE-ITI (GACR P202/12/G061) of the Czech Science Foundation.
} 
of the edge set of the complete graph $K_{N}$ on $N$ vertices there is a homogeneous subset of $n$ vertices, meaning that all edges in the complete subgraph induced by these $n$ vertices have the same color. More generally, for every $k$ and $n$ there exists $N$ such that if the set of all $k$-tuples of elements of an $N$-element set $X$ is colored by two colors, then there exists an $n$-element homogeneous $Y \subseteq X$, with all $k$-tuples from $Y$ having the same color. Let $R_{k}(n)$ stand for the smallest $N$ with this property.

Considering $k$ fixed and $n$ large, the best known lower and upper bounds for the Ramsey function $R_{k}(n)$ are of the form $11 R_{2}(n)=2^{\Theta(n)}$ and, for $k \geq 3$,

$$
\operatorname{twr}_{k-1}\left(\Omega\left(n^{2}\right)\right) \leq R_{k}(n) \leq \operatorname{twr}_{k}(O(n)),
$$

where the tower function $\operatorname{twr}_{k}(x)$ is defined by $\operatorname{twr}_{1}(x)=x$ and $\operatorname{twr}_{i+1}(x)=2^{\operatorname{twr}_{i}(x)}$.

A widely believed, and probably very difficult, conjecture of Erdős and Hajnal asserts that the upper bound is essentially the truth. This is supported by known bounds for more than two colors, where the lower bound for $k$-tuples is also a tower of height $k$; see Conlon, Fox, and Sudakov [CFS13] for a recent improvement and more detailed overview of the known bounds.

Better Ramsey functions for geometric Ramsey-type results. Ramsey's theorem can be used to establish many geometric Ramsey-type results concerning configurations of points, or of other geometric objects, in $\mathbb{R}^{d}$. The first two examples, which up until now remain among the most significant and beautiful ones, come from a 1935 paper of Erdös and Szekeres ES35.

The first one asserts that every sufficiently long sequence $\left(x_{1}, \ldots, x_{N}\right)$ of real numbers contains a subsequence $\left(x_{i_{1}}, x_{i_{2}}, \ldots, x_{i_{n}}\right), i_{1}<i_{2}<\cdots<i_{n}$, that is either increasing, i.e., $x_{i_{1}}<x_{i_{2}}<\cdots<x_{i_{n}}$, or nonincreasing, i.e., $x_{i_{1}} \geq x_{i_{2}} \geq \cdots \geq x_{i_{n}}$.

Ramsey's theorem for $k=2$ yields the bound $N \leq R_{2}(n) \leq \operatorname{twr}_{2}(O(n))$ (color a pair $\{i, j\}$, $i<j$, red if $x_{i}<x_{j}$ and blue if $\left.x_{i} \geq x_{j}\right)$, but the result is known to hold with $N=(n-1)^{2}+1$, an exponential improvement over $R_{2}(n)$.

For the second of the two Erdős-Szekeres theorems mentioned above, we consider a sequence $P=\left(p_{1}, p_{2}, \ldots, p_{N}\right)$ of points in the plane; for simplicity, we assume that the $p_{i}$ are in general position (no three collinear). If $N$ is sufficiently large, then there is a subsequence $\left(p_{i_{1}}, \ldots, p_{i_{n}}\right), i_{1}<i_{2}<\cdots<i_{n}$, forming the vertex set of a convex $n$-gon, enumerated clockwise or counterclockwise.

This time Ramsey's theorem yields $N \leq R_{3}(n) \leq \operatorname{twr}_{3}(O(n))$, by coloring a triple $\{i, j, k\}$, $i<j<k$, red if $p_{i}, p_{j}, p_{k}$ appear clockwise around the boundary of their convex hull, and blue otherwise. Again, the optimal bound is one exponential better, of order $2^{\Theta(n)}$.

It is natural to ask, what is special about the two-colorings of pairs or triples in the above two examples, what makes the Ramsey functions here considerably smaller, compared to arbitrary colorings?

One kind of a combinatorial condition for two-colorings of $k$-tuples implying such improved bounds was given by Fox, Pach, Sudakov, and Suk [FPSS12], and another by the first two authors [EM13; both of them include the two Erdös-Szekeres results as special cases. However, a considerably more general, and probably more interesting, reason for the better

\footnotetext{
${ }^{1}$ We employ the usual asymptotic notation for comparing functions: $f(n)=O(g(n))$ means that $|f(n)| \leq$ $C|g(n)|$ for some $C$ and all $n$, where $C$ may depend on parameters declared as constants (in our case on $k$ ); $f(n)=\Omega(g(n))$ is equivalent to $g(n)=O(f(n))$; and $f(n)=\Theta(g(n))$ means that both $f(n)=O(g(n))$ and $f(n)=\Omega(g(n))$.
} 
Ramsey behavior of these geometric examples is that the colorings are "algebraically defined"; more precisely, they are given by semialgebraic predicates.

Upper bounds for semialgebraic colorings. Let $x_{1}, \ldots, x_{k}$ be points in $\mathbb{R}^{d}$, with $x_{i, j}$ denoting the $j$ th coordinate of $x_{i}$; we regard the $x_{i, j}$ as variables. A $k$-ary d-dimensional semialgebraic predicate $\Phi\left(x_{1}, \ldots, x_{k}\right)$ is a Boolean combination of polynomial equations and inequalities in the $x_{i, j}$. More explicitly, there are a Boolean formula $\phi\left(X_{1}, \ldots, X_{t}\right)$ in Boolean variables $X_{1}, \ldots, X_{t}$ and polynomials $f_{1}, \ldots, f_{t}$ in the variables $x_{i, j}, 1 \leq i \leq k, 1 \leq j \leq d$, such that $\Phi\left(x_{1}, \ldots, x_{k}\right)=\phi\left(A_{1}, \ldots, A_{t}\right)$, where $A_{\ell}$ is true if $f_{\ell}\left(x_{1,1}, \ldots, x_{k, d}\right) \geq 0$ and false otherwise.

We call a sequence $\left(p_{1}, \ldots, p_{n}\right)$ of points in $\mathbb{R}^{d} \Phi$-homogeneous if either $\Phi\left(p_{i_{1}}, \ldots, p_{i_{k}}\right)$ holds for every choice $1 \leq i_{1}<\cdots<i_{k} \leq n$, or it holds for no such choice. The Ramsey function $R_{\Phi}(n)$ is the smallest $N$ such that every point sequence of length $N$ contains a $\Phi$-homogeneous subsequence of length $n$.

The following general upper bound was first proved by Alon, Pach, Pinchasi, Radoičić, and Sharir $\left.\mathrm{APP}^{+} 05\right]$ for $k=2$, and then generalized by Conlon, Fox, Pach, Sudakov, and Suk $\left[\mathrm{CFP}^{+} 13\right]$ for $k \geq 3$ :

Theorem $1.1\left(\left[\mathrm{APP}^{+} 05, \mathrm{CFP}^{+} 13\right]\right)$. For every $d, k$, and a $k$-ary d-dimensional semialgebraic predicate $\Phi$,

$$
R_{\Phi}(n) \leq \operatorname{twr}_{k-1}\left(n^{C}\right),
$$

where $C$ is a constant depending on $d, k, \Phi 2$

Thus, the Ramsey function for $k$-ary semialgebraic predicates is bounded above by a tower one lower than the "combinatorial" Ramsey function $R_{k}(n)$. Let us note that for the case of increasing or nonincreasing subsequences $(k=2, d=1)$ and subsequences in convex position $(k=3, d=2)$ as above, Theorem 1.1 yields somewhat weak bounds, namely, $n^{O(1)}$ and $2^{n^{O(1)}}$ instead of $n^{2}$ and $2^{O(n)}$, respectively, but still in the right range.

By very different methods, Bukh and the second author BM12 obtained a doubly exponential upper bound for all one-dimensional semialgebraic predicates, for arbitrary $k$ :

Theorem 1.2 ([BM12]). For every 1-dimensional semialgebraic predicate $\Phi$ there is a constant $C$ such that $R_{\Phi}(n) \leq \operatorname{twr}_{3}(C n)$.

This opens an interesting possibility, namely, that the Ramsey function of $d$-dimensional semialgebraic predicates might be bounded by a tower whose height depends only on $d$ (and not on $k$ ), but currently this question is wide open. But certainly it makes it interesting to study the dependence of the Ramsey function on the dimension.

Lower bounds. The classical Erdös-Szekeres result on subsequences in convex position ES35] supplies a lower bound of $2^{\Omega(n)}=\operatorname{twr}_{2}(\Omega(n))$ in the setting of Theorem 1.1 for $k=3$ and $d=2$. The first two authors EM13] constructed a reasonably natura 3 4-ary planar

\footnotetext{
${ }^{2}$ Actually, the constant $C$ depends on $\Phi$ only through its description complexity, which Conlon et al. define as $\max (m, D)$, where $m$ is the number of polynomials occurring in $\Phi$ and $D$ is the maximum degree of these polynomials. Thus, the bound does not depend on the magnitude of the coefficients in the polynomials.

${ }^{3}$ By a "natural" predicate we mean here one that has a clear geometric meaning and seems reasonable to study in its own right, not only as a lower-bound example for a general result. In the case of [EM13], assuming that the considered four points $x_{1}, \ldots, x_{4}$ are numbered in the order of increasing first coordinates, the predicate asserts that $x_{4}$ lies above the graph of the unique quadratic polynomial passing through $x_{1}, x_{2}, x_{3}$.
} 
semialgebraic $\Phi$ with $R_{\Phi}(n) \geq \operatorname{twr}_{3}(\Omega(n))$. This shows that for $k \leq 4$, the height of the tower in Theorem 1.1 is optimal in terms of $k$.

For $d=1$, BM12] provided a one-dimensional 5-ary $\Phi$ with $R_{\Phi}(n) \geq \operatorname{twr}_{3}(\Omega(n))$, matching Theorem 1.2. Conlon et al. $\left.\mathrm{CFP}^{+} 13\right]$ improved the arity to 4, which is optimal in view of Theorem 1.1.

Moreover, they obtained a lower bound almost matching Theorem 1.1 for an arbitrary $k$. Namely, for every $k \geq 4$ they constructed a $d$-dimensional $k$-ary semialgebraic predicate $\Phi$ such that $R_{\Phi}(n) \geq \operatorname{twr}_{k-1}(\Omega(n))$. However, the dimension $d$ in their construction is large: $d=2^{k-4}$.

A stronger lower bound. In this paper we first modify (and simplify) the lower bound construction of Conlon et al. [CFP $\left.{ }^{+} 13\right]$, obtaining examples in considerably lower dimension.

Theorem 1.3. For every $d \geq 2$ there is a d-dimensional semialgebraic predicate $\Phi$ of arity $k=d+3$ such that

$$
R_{\Phi}(n) \geq \operatorname{twr}_{k-1}(\Omega(n)) .
$$

The proof is given in Section 2, In view of Theorem 1.2, the dependence of the tower height on the dimension in this result might even be optimal.

Super-order-type homogeneous subsequences. Next, we provide a natural geometric Ramsey-type theorem in $\mathbb{R}^{d}$ in which the Ramsey function is a tower of height $d$.

Let $T=\left(p_{1}, \ldots, p_{d+1}\right)$ be an ordered $(d+1)$-tuple of points in $\mathbb{R}^{d}$. We recall that the sign (or orientation) of $T$ is defined as $\operatorname{sgn} \operatorname{det} M$, where the $j$ th column of the $(d+1) \times(d+1)$ matrix $M$ is $\left(1, p_{j, 1}, p_{j, 2}, \ldots, p_{j, d}\right)$. Geometrically, the sign is +1 if the $d$-tuple of vectors $p_{1}-p_{d+1}, \ldots, p_{d}-p_{d+1}$ forms a positively oriented basis of $\mathbb{R}^{d}$, it is -1 if it forms a negatively oriented basis, and it is 0 if these vectors are linearly dependent.

We call a sequence $\left(p_{1}, p_{2}, \ldots, p_{n}\right)$ of points in $\mathbb{R}^{d}$ in general position order-type homogeneous if all $(d+1)$-tuples $\left(p_{i_{1}}, \ldots, p_{i_{d+1}}\right), i_{1}<\cdots<i_{d+1}$, have the same sign (which is nonzero, by the general position assumption). Such sequences are of interest from various points of view: For example, the convex hull of an order-type homogeneous sequence is combinatorially equivalent to a cyclic polytope (see, e.g., Zie94 for background). They can also be viewed as discrete Chebyshev systems; see [KS66], as well as a remark below.

By Ramsey's theorem, every sufficiently long point sequence in general position contains an order-type homogeneous subsequence of length $n$ (we color every $(d+1)$-tuple by its sign). Letting $\mathrm{OT}_{d}(n)$ be the corresponding Ramsey function, we obtain $\mathrm{OT}_{d}(n) \leq \operatorname{twr}_{d}\left(n^{C}\right)$ from Theorem 1.1. This has recently been improved to $\mathrm{OT}_{d}(n) \leq \operatorname{twr}_{d}(O(n))$ by Suk [Suk13].

This upper bound is essentially tight. Until recently this was proved only for $d=2$ (by [ES35]) and $d=3$ [EM13]. As will be explained next, our results, together with a recent paper of Bárány, Pór, and the second author [BMP13], yield a matching lower bound for all $d$.

In the present paper we prove a lower bound for a somewhat stronger notion of homogeneity. Namely, let $\pi_{j}: \mathbb{R}^{d} \rightarrow \mathbb{R}^{j}$ denote the projection on the first $j$ coordinates. We say that a point sequence $P=\left(p_{1}, \ldots, p_{n}\right)$ in $\mathbb{R}^{d}$ is super-order-type homogeneous if, for each $j=1,2, \ldots, d$, the projected sequence $\pi_{j}(P)=\left(\pi_{j}\left(p_{1}\right), \ldots \pi_{j}\left(p_{n}\right)\right)$ is order-type homogeneous.

By iterated application of Ramsey's theorem, it can be seen that every sufficiently long point sequence in general position in $\mathbb{R}^{d}$ contains a super-order-type homogeneous subsequence of length $n$. Let $\mathrm{OT}_{d}^{*}(n)$ be the corresponding Ramsey function. We have the following lower bound, proved in Section 3 , 
Theorem 1.4. For every $n \geq d+1$ OT $_{d}^{*}(n) \geq \operatorname{twr}_{d}(n-d)$.

In BMP13] it is proved that $\mathrm{OT}_{d}^{*}(n) \leq \mathrm{OT}_{d}\left(C_{d} n\right)$ for every $d$, where $C_{d}$ is a suitable constant. Thus, we also obtain a lower bound for $\mathrm{OT}_{d}$, which is tight up to a multiplicative constant in front of $n$ :

Corollary 1.5. We have $\mathrm{OT}_{d}(n) \geq \operatorname{twr}_{d}(\Omega(n))$.

Chebyshev systems. Let $A$ be a linearly ordered set of at least $k+1$ elements. A (real) Chebyshev system (also spelled Tchebycheff) on $A$ is a system of continuous real functions $f_{0}, f_{1}, \ldots, f_{k}: A \rightarrow \mathbb{R}$ such that for every choice of elements $t_{0}<t_{1}<\cdots<t_{k}$ in $A$, the matrix

$\left(f_{i}\left(t_{j}\right)\right)_{i, j=0}^{k}$ has a (strictly) positive determinant. Chebyshev systems are mostly considered for $A$ an interval in $\mathbb{R}$ with the natural ordering, the basic example being $f_{i}(t)=t^{i}$, but the case of finite $A$ (discrete Chebyshev systems) has been investigated as well. The functions $f_{0}, \ldots, f_{k}$ as above form a Markov system, also called a complete Chebyshev system, if $f_{0}, \ldots, f_{i}$ is a Chebyshev system for every $i=1,2, \ldots, k$. Chebyshev systems are of considerable importance in several areas, such as approximation theory or the theory of finite moments; see the classical monograph of Karlin and Studden [KS66] or, e.g., Carnicer, Peña, and Zalik CPZ98 for a more recent study.

In our setting, it is easy to check that an $n$-point order-type homogeneous sequence $P=$ $\left(p_{1}, \ldots, p_{n}\right)$ in $\mathbb{R}^{d}$ gives rise to a Chebyshev system on $A=\{1,2, \ldots, n\}$, by setting $f_{j}(i)=p_{i, j}$ for $j=1,2, \ldots, d$ and $f_{0} \equiv 1$ (possibly with changing the sign for one of the $f_{i}$, if the signs of the $(d+1)$-tuples in $P$ are negative), and conversely, from a discrete Chebyshev system with $f_{0} \equiv 1$ we obtain an order-type homogeneous sequence. Similarly, super-order-type homogeneous sequences correspond to discrete Markov systems.

\section{Lower bound for semialgebraic predicates in a small dimen- sion}

Here we prove Theorem 1.3. As was remarked in the introduction, our construction can be regarded as a modification of that of Conlon et al. $\mathrm{CFP}^{+} 13$, but we give a self-contained presentation.

Stepping up. The proof proceeds by induction on $d$; having constructed a suitable $d$ dimensional $k$-ary semialgebraic predicate and an $N$-point sequence $P \subset \mathbb{R}^{d}$ without long $\Phi$-homogeneous subsequences, we produce a $(d+1)$-dimensional $(k+1)$-ary semialgebraic predicate $\Psi$ and a $2^{N}$-point sequence $Q \subset \mathbb{R}^{d+1}$ without long $\Psi$-homogeneous subsequences.

Our basic tool is a classical stepping-up lemma of Erdös and Hajnal, see e.g. GRS90] or CFS13. We first recall it in the standard combinatorial setting, and then we will work on transferring it to a semialgebraic setting.

Let $I=[N]:=\{1,2, \ldots, N\}$, and let $\chi:\left(\begin{array}{l}I \\ k\end{array}\right) \rightarrow\{0,1\}$ be a given two-coloring of all $k$-tuples of $I$. Let $J=\{0,1\}^{N}$ be the set of all binary vectors of length $N$ ordered lexicographically.

We define a coloring $\chi^{\prime}:\left(\begin{array}{c}J \\ k+1\end{array}\right) \rightarrow\{0,1\}$ of all $(k+1)$-tuples of $J$. First we introduce a function $\delta: J \times J \rightarrow I$ by

$$
\delta(\boldsymbol{\alpha}, \boldsymbol{\beta})=\min \left\{i \in I: \alpha_{i} \neq \beta_{i}\right\}
$$


For a $(k+1)$-tuple $\left(\boldsymbol{\alpha}_{1}, \ldots, \boldsymbol{\alpha}_{k+1}\right)$ of binary vectors, $\boldsymbol{\alpha}_{1}<_{\text {lex }} \cdots<_{\text {lex }} \boldsymbol{\alpha}_{k+1}$, we write $\delta_{\ell}:=$ $\delta\left(\boldsymbol{\alpha}_{\ell}, \boldsymbol{\alpha}_{\ell+1}\right)$. Then $\chi^{\prime}$, the stepping-up coloring for $\chi$, is given by

$$
\chi^{\prime}\left(\boldsymbol{\alpha}_{1}, \ldots, \boldsymbol{\alpha}_{k+1}\right):= \begin{cases}\chi\left(\delta_{1}, \ldots, \delta_{k}\right) & \text { if } \delta_{1}<\cdots<\delta_{k} \text { or } \delta_{1}>\cdots>\delta_{k} \\ 1 & \text { if } \delta_{1}<\delta_{2}>\delta_{3} \\ 0 & \text { otherwise. }\end{cases}
$$

Now the stepping-up lemma can be stated as follows.

Lemma 2.1 (Stepping-up lemma). If $\chi$ is a two-coloring of the $k$-tuples of $I:=[N]$ under which I has no homogeneous subset of size n, then, under the stepping-up coloring $\chi^{\prime}$, the set $J=\{0,1\}^{N}$ contains no homogeneous subset of size $2 n+k-4$.

The proof is not very complicated and it can be found, e.g., in [CFP ${ }^{+} 13$, or GRS90, Sec. 4.7].

Semialgebraic stepping up. Now let $\Phi$ be a $d$-dimensional $k$-ary semialgebraic predicate, and let $P=\left(p_{1}, \ldots, p_{N}\right)$ be a point sequence in $\mathbb{R}^{d}$ indexed by the set $I=[N]$ as above. Let $\chi=\chi_{\Phi}$ be the coloring of $k$-tuples of $I$ induced by $\Phi$; that is, for $i_{1}<\cdots<i_{k} \in I$, $\chi\left(i_{1}, \ldots, i_{k}\right)$ is 1 or 0 depending on whether $\Phi\left(p_{i_{1}}, \ldots, p_{i_{k}}\right)$ holds or not.

We want to construct a sequence $Q$ in $\mathbb{R}^{d+1}$ indexed by $J=\{0,1\}^{N}$ and a $(d+1)$ dimensional $(k+1)$-ary semialgebraic predicate $\Psi$ such that the coloring induced by $\Psi$ on $\left(\begin{array}{c}J \\ k+1\end{array}\right)$ is exactly the stepping-up coloring $\chi^{\prime}$. For our construction, we need to assume simple additional properties of $\Phi$ and $P$, which we now introduce.

Let $P=\left(p_{1}, \ldots, p_{N}\right)$ be a sequence of points in $\mathbb{R}^{d}$. We call a predicate $\Phi$ robust 4 on $P$ if there is some $\eta>0$ such that $\Phi\left(p_{i_{1}}, \ldots, p_{i_{k}}\right) \Leftrightarrow \Phi\left(p_{i_{1}}^{\prime}, \ldots, p_{i_{k}}^{\prime}\right)$ whenever $1 \leq i_{1}<\cdots<i_{k} \leq N$ and $\left\|p_{i_{j}}-p_{i_{j}}^{\prime}\right\| \leq \eta$ for all $j=1,2, \ldots, k$.

In defining the new predicate $\Psi$, we will also need to use the linear ordering of the points of $P$. We thus say that a binary semialgebraic predicate $\prec$ on $\mathbb{R}^{d}$ is order-inducing for $P$ if $p_{i} \prec p_{j}$ iff $i<j$, for $i, j=1,2, \ldots, N$.

Now we can state our semialgebraic stepping-up lemma.

Proposition 2.2 (Semialgebraic stepping-up). Let $\Phi$ be a d-dimensional $k$-ary semialgebraic predicate and let $\prec$ be a d-dimensional binary semialgebraic predicate. Then there are a $(d+1)$-dimensional $(k+1)$-ary semialgebraic predicate $\Psi$ and a $(d+1)$-dimensional binary semialgebraic predicate $\prec^{\prime}$ with the following property.

Let $P=\left(p_{1}, \ldots, p_{N}\right)$ be a point sequence in $\mathbb{R}^{d}$ such that $\prec$ is order-inducing on $P$ and both $\Phi$ and $\prec$ are robust on $P$, and let $\chi_{\Phi}$ be the coloring of $k$-tuples of $I=[N]$ induced by $\Phi$. Then there is a point sequence $Q=\left(q_{\boldsymbol{\alpha}}: \boldsymbol{\alpha} \in J=\{0,1\}^{N}\right)$ such that $\prec^{\prime}$ is order-inducing on $Q$ (w.r.t. the lexicographic ordering of $J$ ), both $\Psi$ and $\prec^{\prime}$ are robust on $Q$, and the coloring $\chi_{\Psi}$ induced on the $(k+1)$-tuples of $J$ by $\Psi$ is the stepping-up coloring for $\chi_{\Phi}$.

Proof. The construction of $Q$ uses a parameter $\varepsilon>0$, which we assume to be sufficiently small.

For $\boldsymbol{\alpha}=\left(\alpha_{1}, \ldots, \alpha_{N}\right) \in J$, we set

$$
q_{\boldsymbol{\alpha}}:=\sum_{i=1}^{N} \alpha_{i} \varepsilon^{i}\left(1, p_{i, 1}, p_{i, 2}, \ldots, p_{i, d}\right) \in \mathbb{R}^{d+1} .
$$

\footnotetext{
${ }^{4}$ Conlon et al. $\left[\mathrm{CFP}^{+} 13\right.$ use the term $\eta$-deep.
} 
In particular, the first coordinate of $q_{\boldsymbol{\alpha}}$ is $\sum_{i=1}^{N} \alpha_{i} \varepsilon^{i}$. Hence, as is easy to check, for $\varepsilon$ sufficiently small, the lexicographic ordering of $J$ agrees with the ordering of $Q$ by the first coordinate, and hence we can take the standard ordering in the first coordinate as the required order-inducing (and obviously robust) predicate $\prec^{\prime}$ on $Q$.

Next, we define a mapping $\bar{\delta}: \mathbb{R}^{d+1} \times \mathbb{R}^{d+1} \rightarrow \mathbb{R}^{d}$, which will play the role of the $\delta$ from the stepping-up lemma in the geometric setting. For points $x, y \in \mathbb{R}^{d+1}$, we set

$$
\bar{\delta}(x, y):=\left(\frac{x_{2}-y_{2}}{x_{1}-y_{1}}, \frac{x_{3}-y_{3}}{x_{1}-y_{1}}, \ldots, \frac{x_{d+1}-y_{d+1}}{x_{1}-y_{1}}\right) \in \mathbb{R}^{d} .
$$

(Actually, $\bar{\delta}(x, y)$ is undefined for $x_{1}=y_{1}$, but we will use $\bar{\delta}$ only for points with different first coordinates.)

By elementary calculation we can see that for $\boldsymbol{\alpha}, \boldsymbol{\beta} \in J, \boldsymbol{\alpha} \neq \boldsymbol{\beta}$, we have

$$
\lim _{\varepsilon \rightarrow 0} \bar{\delta}\left(q_{\boldsymbol{\alpha}}, q_{\boldsymbol{\beta}}\right)=p_{\delta(\boldsymbol{\alpha}, \boldsymbol{\beta})}
$$

This allows us to imitate the combinatorial definition (1) of the stepping-up coloring by a semialgebraic predicate $\Psi$. For a $(k+1)$-tuple of points $\left(x_{1}, \ldots, x_{k+1}\right)$ in $\mathbb{R}^{d+1}$, let us write $\bar{\delta}_{\ell}:=\bar{\delta}\left(x_{\ell}, x_{\ell+1}\right)$, and set

$$
\Psi\left(x_{1}, \ldots, x_{k+1}\right):= \begin{cases}\Phi\left(\bar{\delta}_{1}, \ldots, \bar{\delta}_{k}\right) & \text { if } \bar{\delta}_{1} \prec \cdots \prec \bar{\delta}_{k} \\ \Phi\left(\bar{\delta}_{k}, \ldots, \bar{\delta}_{1}\right) & \text { if } \bar{\delta}_{1} \succ \cdots \succ \bar{\delta}_{k} \\ \text { true } & \text { if } \bar{\delta}_{1} \prec \bar{\delta}_{2} \succ \bar{\delta}_{3} \\ \text { false } & \text { otherwise. }\end{cases}
$$

As written, $\Psi$ is not necessarily a semialgebraic predicate, since the definition of $\bar{\delta}$ involves division. However, we can always multiply by the denominators and introduce appropriate conditions; e.g., $\frac{u}{v}<1$ can be replaced with $(u<v \wedge v>0) \vee(u>v \wedge v<0)$, which is equivalent whenever $\frac{u}{v}$ is defined. In this way, we obtain an honest semialgebraic predicate.

It remains to check that $\Psi$ induces the stepping-up coloring on $J$, which is straightforward using the robustness of $\Phi$ and $\prec$ and the limit relation (3). Indeed, let us fix $\boldsymbol{\alpha}_{1}<_{\text {lex }} \cdots<_{\text {lex }}$ $\boldsymbol{\alpha}_{k+1} \in J$ and write $\bar{\delta}_{\ell}:=\bar{\delta}\left(q_{\boldsymbol{\alpha}_{\ell}}, q_{\boldsymbol{\alpha}_{\ell+1}}\right)$ and $\delta_{\ell}:=\delta\left(\boldsymbol{\alpha}_{\ell}, \boldsymbol{\alpha}_{\ell+1}\right)$. Then for $\varepsilon$ sufficiently small, we have $\bar{\delta}_{\ell} \prec \bar{\delta}_{\ell+1}$ iff $p_{\delta_{\ell}} \prec p_{\delta_{\ell+1}}$ (by the robustness of $\prec$ ) iff $\delta_{\ell}<\delta_{\ell+1}$ (since $\prec$ is order-inducing on $P$ ). Assuming $\bar{\delta}_{1} \prec \bar{\delta}_{2} \prec \cdots \prec \bar{\delta}_{k}$, we get that $\Phi\left(\bar{\delta}_{1}, \ldots, \bar{\delta}_{k}\right)$ iff $\Phi\left(p_{\delta_{1}}, \ldots, p_{\delta_{k}}\right)$, again for all sufficiently small $\varepsilon$; similarly if $\bar{\delta}_{1} \succ \bar{\delta}_{2} \succ \cdots \succ \bar{\delta}_{k}$. Therefore, the coloring induced by $\Psi$ on $Q$ is indeed the stepping-up coloring for $\chi_{\Phi}$ as claimed.

It remains to verify that $\Psi$ is robust on $Q$, but this is clear from the robustness of $\Phi$ and $\prec$ and the continuity of $\bar{\delta}$ on the subset of $\mathbb{R}^{d+1} \times \mathbb{R}^{d+1}$ where it is defined.

Proof of Theorem 1.3. As announced, we prove the theorem by induction on $d$.

For the base case $d=1$, we use a result of Conlon et al. $\mathrm{CFP}^{+} 13$, who construct a 4 -ary semialgebraic predicate $\Phi_{1}$ on $\mathbb{R}^{1}$ and, for every $n$, a sequence $P_{1} \subset \mathbb{R}$ of length $\operatorname{twr}_{3}(\Omega(n))$ with no $\Psi_{1}$-homogeneous subsequence of length $n$. It is obvious from their construction that $\Psi_{1}$ is robust on $P_{1}$ and that $<$, the usual inequality among real numbers, is robust and order-inducing on $P_{1}$.

The theorem then follows by a $(d-1)$-fold application of Proposition 2.2 together with the stepping-up lemma (Lemma 2.1). 


\section{Lower bound for super order type}

Here we prove Theorem 1.4. Thus, we need to exhibit long point sequences without superorder-type homogeneous subsequences of length $n$. The construction is almost identical to the one in the previous section, only the base case for $d=1$ is different. The proof essentially consists in relating super-order-type homogeneity to another property, which we call supermonotonicity; checking that the constructed sequence has no super-monotone subsequences of length $n$ is straightforward.

First, for convenience, we extend the definition of the bivariate function $\bar{\delta}$ from (2) in the previous section to an arbitrary number of arguments. Namely, we set $\bar{\delta}(p)=p$ and, for $k \geq 2$,

$$
\bar{\delta}\left(p_{1}, \ldots, p_{k+1}\right):=\bar{\delta}\left(\bar{\delta}\left(p_{1}, \ldots, p_{k}\right), \bar{\delta}\left(p_{2}, \ldots, p_{k+1}\right)\right) .
$$

Again, we are going to use $\bar{\delta}$ only with arguments for which it is well defined.

For points $p, q \in \mathbb{R}^{d}$, we write $p<_{1} q$ if $p_{1}<q_{1}$ (strict inequality in the first coordinate). A point sequence $P=\left(p_{1} \ldots, p_{n}\right)$ in $\mathbb{R}^{d}$ is super-monotone if each of the point sequences $\left(\bar{\delta}\left(p_{1}, \ldots, p_{j}\right), \ldots, \bar{\delta}\left(p_{n-j+1}, \ldots, p_{n}\right)\right)$ in $\mathbb{R}^{d-j+1}$ is monotone according to $<_{1}, 1 \leq j \leq d$.

Here is the key technical result.

Proposition 3.1. A point sequence $\left(p_{1}, \ldots, p_{n}\right)$ in $\mathbb{R}^{d}$ is super-monotone if and only if it is super-order-type homogeneous.

The proof will be given at the end of this section, after some algebraic lemmas. First we finish the proof of Theorem 1.4, assuming the proposition.

Proof of Theorem 1.4. We will construct a sequence $P_{d}(n)$ in general position in $\mathbb{R}^{d}$ of length $\operatorname{twr}_{d}(n-d)$ and containing no super-order-type homogeneous subsequence of length $n$.

We proceed by induction on $d$. The inductive hypothesis will include the assumption that the first coordinate in $P_{d}(n)$ is strictly increasing.

For $d=1$ we set $P_{1}(n):=(1,2, \ldots, n-1)$.

Now we construct $P_{d+1}(n)$ from $P_{d}(n-1)=\left(p_{1}, \ldots, p_{N}\right)$, using the same construction as in Proposition 2.2. That is, $P_{d+1}(n)=\left(q_{\boldsymbol{\alpha}}: \boldsymbol{\alpha} \in\{0,1\}^{N}\right)$, where the binary vectors $\boldsymbol{\alpha}$ are ordered lexicographically, and where, with $\varepsilon>0$ sufficiently small,

$$
q_{\boldsymbol{\alpha}}:=\sum_{i=1}^{N} \alpha_{i} \varepsilon^{i}\left(1, p_{i, 1}, p_{i, 2}, \ldots, p_{i, d}\right) \in \mathbb{R}^{d+1}, \quad \boldsymbol{\alpha} \in\{0,1\}^{N} .
$$

(The $\varepsilon$ is different in each inductive step, and in particular, the one used to construct $P_{d+1}(n)$ from $P_{d}(n-1)$ is much smaller than the one used to construct $P_{d}(n-1)$ from $P_{d-1}(n-2)$, etc.) Because of the robustness of the super-order-type condition, we can slightly perturb the points so that they are in general position. As in the previous section, the points of $P_{d+1}(n)$, ordered according to the lexicographic ordering of the indices $\boldsymbol{\alpha}$, have increasing first coordinates (for $\varepsilon$ sufficiently small).

Now we assume for contradiction that $P_{d+1}(n)$ contains a super-order-type homogeneous subsequence $S=\left(s_{1}, \ldots, s_{n}\right)$. By Proposition 3.1. $S$ is super-monotone. Thus, setting $t_{\ell}=$ $\bar{\delta}\left(s_{\ell}, s_{\ell+1}\right), \ell=1,2, \ldots, n-1$, the sequence $T=\left(t_{1}, \ldots, t_{n-1}\right)$ is super-monotone as well by definition.

By the limit relation (3), for $\varepsilon \rightarrow 0$, each $t_{\ell}$ tends to a point $p_{i_{\ell}}$ of $P_{d}(n-1)$. Moreover, by super-monotonicity, we have $t_{1}<_{1} \cdots<_{1} t_{n-1}$. Hence $p_{i_{1}}<_{1} \cdots<_{1} p_{i_{n-1}}$ for sufficiently small 
$\varepsilon$ and therefore, since the first coordinates are increasing in $P_{d}(n-1)$ by the inductive hypothesis, we have $i_{1}<\cdots<i_{n-1}$. Consequently, using Proposition 3.1 again, $\left(p_{i_{1}}, \ldots, p_{i_{n-1}}\right)$ is a super-order-type homogeneous subsequence of $P_{d}(n-1)$ - a contradiction proving the theorem.

Algebraic lemmas. It remains to prove Proposition 3.1, and for this, we need to develop some algebraic results.

Given a $k$-tuple $T=\left(p_{1}, \ldots, p_{k}\right)$ of points in $\mathbb{R}^{d}, 1 \leq k \leq d$, and an index $j \geq k-1$, we put

$$
D_{j}(T)=\operatorname{det}\left(\begin{array}{cccc}
1 & 1 & \cdots & 1 \\
p_{1,1} & p_{2,1} & \cdots & p_{k, 1} \\
\vdots & \vdots & \ddots & \vdots \\
p_{1, k-2} & p_{2, k-2} & \cdots & p_{k, k-2} \\
p_{1, j} & p_{2, j} & \cdots & p_{k, j}
\end{array}\right)
$$

and

$$
\vec{D}_{j}(T)=\left(D_{j}(T), D_{j+1}(T), \ldots, D_{d}(T)\right) .
$$

Let us remark that $k$ is not represented explicitly in the notation, but it can be inferred from the number of arguments of $D_{j}$. We also note that $\operatorname{sgn} D_{k-1}\left(p_{1}, \ldots, p_{k}\right)$ is the sign of the $k$-tuple $\pi_{k-1}(T)$.

Lemma 3.2. If $A=\left(p_{1}, \ldots, p_{k}\right)$ and $B=\left(p_{2}, \ldots, p_{k+1}\right)$, then, for $j \geq k$, we have

$$
D_{k-1}(A) D_{j}(B)-D_{k-1}(B) D_{j}(A)=D_{k-2}\left(p_{2}, \ldots, p_{k}\right) D_{j}\left(p_{1}, \ldots, p_{k+1}\right) .
$$

Proof. It is enough to do the case $j=k$ (we have $j \geq k$, and so in the identity of the lemma, the $j$ th coordinates of the $p_{i}$ appear only in the determinants $D_{j}(A), D_{j}(B)$, and $\left.D_{j}\left(p_{1}, \ldots, p_{k+1}\right)\right)$. We define the $(k+1) \times(k+1)$ matrix

$$
M_{k+1}\left(p_{1}, \ldots, p_{k+1}\right)=\left(\begin{array}{cccc}
1 & 1 & \ldots & 1 \\
p_{1,1} & p_{2,1} & \cdots & p_{k+1,1} \\
\vdots & \vdots & \ddots & \vdots \\
p_{1, k} & p_{2, k} & \cdots & p_{k+1, k}
\end{array}\right) .
$$

All the determinants we are interested in are submatrices of $M_{k+1}$ and they all contain the matrix $M_{k-1}=M_{k-1}\left(p_{2}, \ldots, p_{k}\right)$ associated with $D_{k-2}\left(p_{2}, \ldots, p_{k}\right)$. We can use elementary row and column operations on $M_{k+1}$ to diagonalize $M_{k-1}$ while leaving the determinants fixed, and we can also assume that the entries below $M_{k-1}$, as well as those to the left and to the right of it, are 0 , as is illustrated next:

$$
\left(\begin{array}{c|ccc|c}
1 & 1 & \ldots & 1 & 1 \\
p_{1,1} & p_{2,1} & \ldots & p_{k, 1} & p_{k+1,1} \\
\vdots & \vdots & M_{k-1} & \vdots & \vdots \\
p_{1, k-2} & p_{2, k-2} & \ldots & p_{k, k-2} & p_{k+1, k-2} \\
p_{1, k-1} & p_{2, k-1} & \ldots & p_{k+1, k-1} & p_{k+1, k-1} \\
p_{1, k} & p_{2, k} & \ldots & p_{k+1, k} & p_{k+1, k}
\end{array}\right) \rightarrow\left(\begin{array}{ccccc|c}
0 & m_{1} & \cdots & 0 & 0 \\
0 & 0 & \ldots & 0 & 0 \\
\vdots & \vdots & \ddots & \vdots & \vdots \\
0 & 0 & \ldots & m_{k-2} & 0 \\
x & 0 & \ldots & 0 & u \\
y & 0 & \ldots & 0 & v
\end{array}\right) .
$$


Now we can compute the determinants in the following way:

$$
\begin{aligned}
D_{k}\left(p_{1}, \ldots, p_{k+1}\right) & =(-1)^{k+1}(x v-y u) \operatorname{det}\left(M_{k-1}\right) & D_{k}(B) & =v \operatorname{det}\left(M_{k-1}\right) \\
D_{k-2}\left(p_{2}, \ldots, p_{k}\right) & =\operatorname{det}\left(M_{k-1}\right) & D_{k}(A) & =(-1)^{k+1} y \operatorname{det}\left(M_{k-1}\right) \\
D_{k-1}(A) & =(-1)^{k+1} x \operatorname{det}\left(M_{k-1}\right) & D_{k-1}(B) & =u \operatorname{det}\left(M_{k-1}\right) .
\end{aligned}
$$

The lemma follows.

\section{Lemma 3.3.}

$$
\bar{\delta}\left(p_{1}, \ldots, p_{k}\right)=\frac{\vec{D}_{k}\left(p_{1}, \ldots, p_{k}\right)}{D_{k-1}\left(p_{1}, \ldots, p_{k}\right)} .
$$

Proof. The proof goes by induction on $k$. The cases $k=1,2$ are trivial. Assume the lemma is true for $k$ and we have points $p_{1}, \ldots, p_{k+1} \in \mathbb{R}^{d}$. For simplicity we write $A=\left(p_{1}, \ldots, p_{k}\right)$ and $B=\left(p_{2}, \ldots, p_{k+1}\right)$. Then we have, with $\pi: \mathbb{R}^{d} \rightarrow \mathbb{R}^{d-1}$ denoting the projection omitting the first coordinate,

$$
\begin{aligned}
\bar{\delta}\left(p_{1}, \ldots, p_{k+1}\right) & =\bar{\delta}(\bar{\delta}(A), \bar{\delta}(B)) \\
& =\frac{\pi(\bar{\delta}(A))-\pi(\bar{\delta}(B))}{(\bar{\delta}(A))_{1}-(\bar{\delta}(B))_{1}} \\
& =\frac{D_{k-1}(A) \vec{D}_{k+1}(B)-D_{k-1}(B) \vec{D}_{k+1}(A)}{D_{k-1}(A) D_{k}(B)-D_{k-1}(B) D_{k}(A)} .
\end{aligned}
$$

The last equality follows from the fact that $\pi\left(\vec{D}_{k}\right)=\vec{D}_{k+1}$ and by clearing the denominators. To finish the proof we use Lemma 3.2 on the denominator and each coordinate of the numerator.

Proof of Proposition 3.1. We generalize the notions of super-monotonicity and superorder-type homogeneity as follows. We say that a point sequence $\left(p_{1} \ldots, p_{n}\right)$ is $k$-monotone if for all $j \leq k$ the point sequence $\left(\bar{\delta}\left(p_{1}, \ldots, p_{j}\right), \ldots, \bar{\delta}\left(p_{n-j+1}, \ldots, p_{n}\right)\right)$ is monotone according to $<_{1}$. We say that $\left(p_{1} \ldots, p_{n}\right)$ is $k$-order-type homogeneous if for all $j \leq k$ the sequence of projections $\left(\pi_{j}\left(p_{1}\right) \ldots, \pi_{j}\left(p_{n}\right)\right)$ in $\mathbb{R}^{j}$ is order-type homogeneous.

By induction on $k$, we prove that a point sequence $\left(p_{1}, \ldots, p_{n}\right)$ in $\mathbb{R}^{d}$ is $k$-monotone if and only if it is $k$-order-type homogeneous; for $k=d$ this is the statement of the proposition.

The cases $k=1,2$ are trivial. So we assume that the claim is true up to some $k$ and we are given a sequence of $n$ points. We may assume that this sequence is $k$-monotone, and hence also $k$-order-type homogeneous. Then we only need to show that $\left(\bar{\delta}\left(p_{1}, \ldots, p_{k+1}\right), \ldots, \bar{\delta}\left(p_{n-k}, \ldots, p_{n}\right)\right)$ is order-type homogeneous iff $\left(\pi_{k+1}\left(p_{1}\right), \ldots, \pi_{k+1}\left(p_{n}\right)\right)$ is monotone according to $<_{1}$.

Let $\left(q_{1}, \ldots, q_{k+2}\right)$ be a $(k+2)$-point subsequence of $\left(p_{1}, \ldots, p_{n}\right)$. By Lemma 3.3, the condition $\bar{\delta}\left(q_{1}, \ldots, q_{k+1}\right)<_{1} \bar{\delta}\left(q_{2}, \ldots, q_{k+2}\right)$ is equivalent to

$$
\frac{D_{k+1}\left(q_{2}, \ldots, q_{k+2}\right)}{D_{k}\left(q_{2}, \ldots, q_{k+2}\right)}-\frac{D_{k+1}\left(q_{1}, \ldots, q_{k+1}\right)}{D_{k}\left(q_{1}, \ldots, q_{k+1}\right)}>0 .
$$

Since $\left(q_{1}, \ldots, q_{k+2}\right)$ is $k$-order-type homogeneous, we have

$$
D_{k}\left(q_{1}, \ldots, q_{k+1}\right) D_{k}\left(q_{2}, \ldots, q_{k+2}\right)>0,
$$


and therefore, (4) is equivalent to

$$
D_{k+1}\left(q_{2}, \ldots, q_{k+2}\right) D_{k}\left(q_{1}, \ldots, q_{k+1}\right)-D_{k+1}\left(q_{1}, \ldots, q_{k+1}\right) D_{k}\left(q_{2}, \ldots, q_{k+2}\right)>0 .
$$

By Lemma 3.2 this is just

$$
D_{k-1}\left(q_{2} \ldots, q_{k+1}\right) D_{k+1}\left(q_{1} \ldots, q_{k+2}\right)>0 .
$$

Since our sequence is also $(k-1)$-order-type homogeneous, the numbers

$$
D_{k-1}\left(p_{1} \ldots, p_{k}\right), D_{k-1}\left(p_{2} \ldots, p_{k+1}\right), \ldots, D_{k-1}\left(p_{n-k+1} \ldots, p_{n}\right)
$$

have the same sign and therefore the numbers

$$
D_{k+1}\left(p_{1} \ldots, p_{k+2}\right), D_{k+1}\left(p_{2} \ldots, p_{k+3}\right), \ldots, D_{k+1}\left(p_{n-k-1} \ldots, p_{n}\right)
$$

also have the same sign. This is precisely the condition needed for the sequence to be $(k+1)-$ order-type homogeneous.

\section{Acknowledgment}

We would like to thank Imre Bárány for useful discussions.

\section{References}

$\left[\mathrm{APP}^{+} 05\right]$ N. Alon, J. Pach, R. Pinchasi, R. Radoičić, and M. Sharir. Crossing patterns of semi-algebraic sets. J. Comb. Theory, Ser. A, 111(2):310-326, 2005.

[BM12] B. Bukh and J. Matoušek. Erdős-Szekeres-type statements: Ramsey function and decidability in dimension 1. Preprint, arXiv:1207.0705, 2012.

[BMP13] I. Bárány, J. Matoušek, and A. Pór. Curves in $\mathbb{R}^{d}$ intersecting every hyperplane at most d+1 times. Preprint, arXiv:1309.1147, 2013.

$\left[\mathrm{CFP}^{+} 13\right]$ D. Conlon, J. Fox, J. Pach, B. Sudakov, and A. Suk. Ramsey-type results for semi-algebraic relations. Trans. Amer. Math. Soc., 2013. To appear. Preprint arXiv:1301.0074. Extended abstract in Proc. 29th Annual ACM Symposium on Computational Geometry, Rio de Janeiro, Brazil, 2013.

[CFS13] D. Conlon, J. Fox, and B. Sudakov. An improved bound for the stepping-up lemma. Discrete Applied Mathematics, 161:1191-1196, 2013.

[CPZ98] J.M. Carnicer, J.M. Peña, and R.A. Zalik. Strictly totally positive systems. J. Approximation Theory, 92(3):411-441, 1998.

[EM13] M. Eliáš and J. Matoušek. Higher-order Erdős-Szekeres theorems. Advances in Mathematics, 244(0):1-15, 2013.

[ES35] P. Erdős and G. Szekeres. A combinatorial problem in geometry. Compositio Math., 2:463-470, 1935. 
[FPSS12] J. Fox, J. Pach, B. Sudakov, and A. Suk. Erdős-Szekeres-type theorem for monotone paths and convex bodies. Proceedings of the London Mathematical Society, 105(5):953-982, 2012.

[GRS90] R. L. Graham, B. L. Rothschild, and J. Spencer. Ramsey Theory. J. Wiley \& Sons, New York, 1990.

[KS66] S. Karlin and W.J. Studden. Tchebycheff systems: With applications in analysis and statistics. Pure and Applied Mathematics, Vol. XV. Interscience Publishers John Wiley \& Sons, New York-London-Sydney, 1966.

[Suk13] A. Suk. A note on order-type homogeneous point sets. Arxiv preprint 1305.5934, 2013.

[Zie94] G. M. Ziegler. Lectures on Polytopes, volume 152 of Graduate Texts in Mathematics. Springer-Verlag, Heidelberg, 1994. Corrected and revised printing 1998. 